\title{
Structure-Preserving Interpolation for Model Reduction of Parametric Bilinear Systems
}

\author{
Peter Benner* Serkan Gugercin ${ }^{\dagger} \quad$ Steffen W. R. Werner ${ }^{\ddagger}$
}

\begin{abstract}
In this paper, we present an interpolation framework for structure-preserving model order reduction of parametric bilinear dynamical systems. We introduce a general setting, covering a broad variety of different structures for parametric bilinear systems, and then provide conditions on projection spaces for the interpolation of structured subsystem transfer functions such that the system structure and parameter dependencies are preserved in the reduced-order model. Two benchmark examples with different parameter dependencies are used to demonstrate the theoretical analysis.
\end{abstract}

Keywords: model order reduction, parametric bilinear systems, moment matching, structure-preserving approximation, structured parametric interpolation

\section{Introduction}

Design and control processes usually involve simulating systems of differential equations describing the underlying dynamics. In the setting of nonlinear or stochastic processes, an important class of such systems are parametric bilinear time-invariant systems; see, e.g., [1,14, 15, 18] for some applications of bilinear systems. In most cases, these bilinear systems have special structures resulting from the underlying physical model and the dynamics are parameter dependent. For example, in case of parametric bilinear mechanical systems, they have the form

$$
\begin{aligned}
& M(\mu) \ddot{x}(t ; \mu)+D(\mu) \dot{x}(t ; \mu)+K(\mu) x(t ; \mu)=B_{\mathrm{u}}(\mu) u(t) \\
& \quad+\sum_{j=1}^{m} N_{\mathrm{p}, j}(\mu) x(t ; \mu) u_{j}(t)+\sum_{j=1}^{m} N_{\mathrm{v}, j}(\mu) \dot{x}(t ; \mu) u_{j}(t), \\
& y(t ; \mu)=C_{\mathrm{p}}(\mu) x(t ; \mu)+C_{\mathrm{v}}(\mu) \dot{x}(t ; \mu),
\end{aligned}
$$

where $M(\mu), D(\mu), K(\mu), N_{\mathrm{p}, j}(\mu), N_{\mathrm{v}, j}(\mu) \in \mathbb{R}^{n \times n}$, for $j=1, \ldots, m ; B_{\mathrm{u}}(\mu) \in \mathbb{R}^{n \times m}$ and $C_{\mathrm{p}}(\mu), C_{\mathrm{v}}(\mu) \in \mathbb{R}^{p \times n}$

\footnotetext{
* Max Planck Institute for Dynamics of Complex Technical Systems, Sandtorstr. 1, 39106 Magdeburg, Germany.

E-mail: benner@mpi-magdeburg.mpg.de

Otto von Guericke University, Faculty of Mathematics, Univer-

sitätsplatz 2, 39106 Magdeburg, Germany.

E-mail: peter.benner@ovgu.de

ORCID: 0000-0003-3362-4103

${ }^{\dagger}$ Department of Mathematics and Computational Modeling and Data Analytics Division, Academy of Integrated Science, Virginia Tech, Blacksburg, VA 24061, USA.

E-mail: gugercin@vt.edu

ORCID: 0000-0003-4564-5999

‡Max Planck Institute for Dynamics of Complex Technical Systems, Sandtorstr. 1, 39106 Magdeburg, Germany.

E-mail: werner@mpi-magdeburg.mpg.de

ORCID: 0000-0003-1667-4862
}

are constant matrices; and $\mu \in \mathbb{M} \subset \mathbb{R}^{d}$ represents the (constant) parameters affecting the dynamics. In (1),

$$
u(t)=\left[u_{1}(t), \quad u_{2}(t), \quad \ldots, \quad u_{m}(t)\right]^{\top} \in \mathbb{R}^{m}
$$

denotes the inputs (forcing), $y(t ; \mu) \in \mathbb{R}^{p}$ the outputs (measurements), and $x(t ; \mu) \in \mathbb{R}^{n \times n}$ the internal variables. The parameter $\mu$ may represent variations in, e.g., material properties or system geometry.

Due to an increasing demand for accuracy in the modeling stage, systems as in (1) become larger and larger, e.g., $n>10^{6}$, imposing overwhelming demands on computational resources like time and memory. The situation is even more prominent in the parametric problems we consider here due to the need to evaluate/simulate (1) for many samples of $\mu$. The aim of parametric model order reduction is to construct a cheap-to-evaluate approximation of the input-to-output behavior of the original system by reducing the state-space dimension, i.e., the number of equations $n$, in such a way that the reduced model provides a high-fidelity approximation to the original one for the parameter range of interest. Additionally, the reduced-order model should have the same internal structure as well as the parameter dependencies as the original to retain the underlying physical structure. For example, for the system (1), the structure-preserving parametric reduced-order model will have the form

$$
\begin{aligned}
& \widehat{M}(\mu) \ddot{\hat{x}}(t ; \mu)+\widehat{D}(\mu) \dot{\hat{x}}(t ; \mu)+\widehat{K}(\mu) \hat{x}(t ; \mu)=\widehat{B}_{\mathrm{u}}(\mu) u(t) \\
& \quad+\sum_{j=1}^{m} \widehat{N}_{\mathrm{p}, j}(\mu) \hat{x}(t ; \mu) u_{j}(t)+\sum_{j=1}^{m} \widehat{N}_{\mathrm{v}, j}(\mu) \dot{\hat{x}}(t ; \mu) u_{j}(t), \quad(2) \\
& \qquad \hat{y}(t ; \mu)=\widehat{C}_{\mathrm{p}}(\mu) \hat{x}(t ; \mu)+\widehat{C}_{\mathrm{v}}(\mu) \dot{\hat{x}}(t ; \mu), \\
& \text { with } \widehat{M}(\mu), \widehat{D}(\mu), \widehat{K}(\mu), \widehat{N}_{\mathrm{p}, j}(\mu), \widehat{N}_{\mathrm{v}, j}(\mu) \in \mathbb{R}^{r \times r}, \text { for } j=
\end{aligned}
$$


$1, \ldots, m, \widehat{B}_{\mathrm{u}}(\mu) \in \mathbb{R}^{r \times m}, \widehat{C}_{\mathrm{p}}(\mu), \widehat{C}_{\mathrm{v}}(\mu) \in \mathbb{R}^{p \times r}$, and $r \ll n$. Note that the reduced-order model (2) has the same structure as (1) and can be interpreted as a physically meaningful reduced-order mechanical system. The structure preservation can also be very beneficial in terms of computational speed and accuracy; see, e.g., [8,9].

For parametric unstructured (classical) bilinear systems, i.e., for systems of the form

$$
\begin{aligned}
E(\mu) \dot{x}(t ; \mu)= & A(\mu) x(t ; \mu)+B(\mu) u(t) \\
& +\sum_{j=1}^{m} N_{j}(\mu) x(t ; \mu) u_{j}(t), \\
y(t ; \mu)= & C(\mu) x(t ; \mu),
\end{aligned}
$$

the interpolatory parametric model reduction framework was developed in [16] by synthesizing the interpolation theory for parametric linear dynamical systems [3,5] with the subsystem interpolation approaches for bilinear systems [3,4,10-12]. Recently in [8], the structured interpolation framework of [7] for linear dynamical systems has been extended to the case of structured bilinear systems for non-parametric structured bilinear systems. In this paper, we will extend this interpolation theory to the case of structured parametric bilinear systems.

In Section 2, we introduce the basic mathematical concepts and notation. We prove the structure-preserving interpolation framework for parametric bilinear systems in Section 3. The established theory is then extended in Section 4 to the interpolation of parameter sensitivities. Section 5 illustrates the analysis in two numerical benchmark examples, followed by conclusions in Section 6 .

\section{Mathematical preliminaries}

Under some mild assumptions, the output of the bilinear system (3) can be rewritten in terms of a Volterra series, i.e.,

$$
\begin{aligned}
y(t ; \mu)= & \sum_{k=1}^{\infty} \int_{0}^{t} \int_{0}^{t_{1}} \ldots \int_{0}^{t_{k-1}} g_{k}\left(t_{1}, \ldots, t_{k}, \mu\right) \\
& \times\left(u\left(t-\sum_{i=1}^{k} t_{i}\right) \otimes \cdots \otimes u\left(t-t_{1}\right)\right) \mathrm{d} t_{k} \cdots \mathrm{d} t_{1},
\end{aligned}
$$

where $g_{k}$ denotes the $k$-th regular Volterra kernel; see, e.g., [17]. Using the multivariate Laplace transformation [17], the regular Volterra kernels yield the frequency representation (4), as the $k$-th regular transfer function of $(3)$, where $N(\mu)=\left[N_{1}(\mu), \ldots, N_{m}(\mu)\right]$. The model reduction theory in [16] is based on the interpolation of (4), i.e., unstructured (classical) parametric subsystems.

In this paper, we consider a much more general setting of multivariate transfer functions. The interpolation of structured transfer functions for linear systems was developed in [7] and then extended to the parametric setting in [2]. As the structured transfer functions were recently extended to non-parametric bilinear systems in [8], we consider here structured parametric multivariate transfer functions of the form (5) with frequency points $s_{1}, \ldots, s_{k} \in \mathbb{C}$, constant parameters $\mu \in \mathbb{M} \subset \mathbb{R}^{d}$, $\mathcal{N}(s, \mu)=\left[\mathcal{N}_{1}(s, \mu), \quad \ldots, \quad \mathcal{N}_{m}(s, \mu)\right]$, and matrix functions

$$
\begin{gathered}
\mathcal{C}: \mathbb{C} \times \mathbb{M} \rightarrow \mathbb{C}^{p \times n}, \quad \mathcal{K}: \mathbb{C} \times \mathbb{M} \rightarrow \mathbb{C}^{n \times n}, \\
\mathcal{B}: \mathbb{C} \times \mathbb{M} \rightarrow \mathbb{C}^{n \times m}, \quad \mathcal{N}_{j}: \mathbb{C} \times \mathbb{M} \rightarrow \mathbb{C}^{n \times n},
\end{gathered}
$$

for $j=1, \ldots, m$. For the parametric bilinear mechanical systems (1), these matrix functions are realized by

$$
\begin{aligned}
\mathcal{K}(s, \mu) & =s^{2} M(\mu)+s D(\mu)+K(\mu), \\
\mathcal{N}_{j}(s, \mu) & =N_{\mathrm{p}, j}(\mu)+s N_{\mathrm{v}, j}(\mu) \text { for } j=1, \ldots, m, \\
\mathcal{B}(s, \mu) & =B_{\mathrm{u}}, \text { and } \mathcal{C}(s, \mu)=C_{\mathrm{p}}(\mu)+s C_{\mathrm{v}}(\mu) .
\end{aligned}
$$

The reduced-order models are then computed by projection: given model reduction bases $V, W \in \mathbb{C}^{n \times r}$, the reduced-order model $\widehat{G}$ is described by the reduced-order matrix functions

$$
\begin{aligned}
\widehat{\mathcal{C}}(s, \mu) & =\mathcal{C}(s, \mu) V, & \widehat{\mathcal{K}}(s, \mu) & =W^{\mathrm{H}} \mathcal{K}(s, \mu) V, \\
\widehat{\mathcal{B}}(s, \mu) & =W^{\mathrm{H}} \mathcal{B}(s, \mu), & \widehat{\mathcal{N}}_{j}(s, \mu) & =W^{\mathrm{H}} \mathcal{N}_{j}(s, \mu) V,
\end{aligned}
$$

for $j=1, \ldots, m$. In general, every matrix-valued function can be affinely decomposed with respect to its arguments and we can write

$$
\mathcal{K}(s, \mu)=\sum_{j=1}^{n_{\mathcal{K}}} h_{\mathcal{K}, j}(s, \mu) \mathcal{K}_{j},
$$

where $h_{\mathcal{K}, j}: \mathbb{C} \times \mathbb{M} \rightarrow \mathbb{C}$ are scalar functions depending on frequency and parameter, and $\mathcal{K}_{j} \in \mathbb{C}^{n \times n}$ are constant matrices, for $j=1, \ldots, n_{\mathcal{K}}$. In the worst-case scenario, we have $n_{\mathcal{K}}=n^{2}$ and $\mathcal{K}_{j}$ 's are the elementary matrices. However, we are interested in cases where $n_{\mathcal{K}}$ is modest, which is the case in most applications. Using the affine decomposition, the reduced-order matrix function is then given by

$$
\begin{aligned}
\widehat{\mathcal{K}}(s, \mu) & =W^{\mathrm{H}} \mathcal{K}(s, \mu) V=\sum_{j=1}^{n_{\mathcal{K}}} h_{\mathcal{K}, j}(s, \mu) W^{\mathrm{H}} \mathcal{K}_{j} V \\
& =\sum_{j=1}^{n_{\mathcal{K}}} h_{\mathcal{K}, j}(s, \mu) \widehat{\mathcal{K}}_{j} .
\end{aligned}
$$

This works analogously for the other matrix functions in (7), which gives a computable realization of the reduced-order model. Since the functions $h_{\mathcal{K}, j}$ stay unchanged, the internal structure and parameter dependency of the original matrix functions, (and thus of the original system) are retained.

In the following, we will use an abbreviation for the notion of partial derivatives, namely we denote

$$
\partial_{s_{1}^{j_{1} \ldots s_{k} j_{k}}} f\left(z_{1}, \ldots, z_{k}\right):=\frac{\partial^{j_{1}+\ldots+j_{k}} f}{\partial s_{1}^{j_{1}} \cdots \partial s_{k}^{j_{k}}}\left(t_{1}, \ldots, t_{k}\right),
$$




$$
\begin{aligned}
& G_{k}\left(s_{1}, \ldots, s_{k}, \mu\right)=C(\mu)\left(s_{k} E(\mu)-A(\mu)\right)^{-1}\left(\prod_{j=1}^{k-1}\left(I_{m^{j-1}} \otimes N(\mu)\right)\left(I_{m^{j}} \otimes\left(s_{k-j} E(\mu)-A(\mu)\right)^{-1}\right)\right)\left(I_{m^{k-1}} \otimes B(\mu)\right), \quad k \geq 1 \\
& G_{k}\left(s_{1}, \ldots, s_{k}, \mu\right)=\mathcal{C}\left(s_{k}, \mu\right) \mathcal{K}\left(s_{k}, \mu\right)^{-1}\left(\prod_{j=1}^{k-1}\left(I_{m^{j-1}} \otimes \mathcal{N}\left(s_{k-j}, \mu\right)\right)\left(I_{m^{j}} \otimes \mathcal{K}\left(s_{k-j}, \mu\right)^{-1}\right)\right)\left(I_{m^{k-1}} \otimes \mathcal{B}\left(s_{1}, \mu\right)\right), \quad k \geq 1 \\
& \widehat{G}_{k}\left(s_{1}, \ldots, s_{k}, \mu\right)=\widehat{\mathcal{C}}\left(s_{k}, \mu\right) \widehat{\mathcal{K}}\left(s_{k}, \mu\right)^{-1}\left(\prod_{j=1}^{k-1}\left(I_{m^{j-1}} \otimes \widehat{\mathcal{N}}\left(s_{k-j}, \mu\right)\right)\left(I_{m^{j}} \otimes \widehat{\mathcal{K}}\left(s_{k-j}, \mu\right)^{-1}\right)\right)\left(I_{m^{k-1}} \otimes \widehat{\mathcal{B}}\left(s_{1}, \mu\right)\right), \quad k \geq 1
\end{aligned}
$$

for the differentiation of an analytic function $f: \mathbb{C}^{k} \rightarrow \mathbb{C}^{\ell}$ with respect to the variables $s_{1}, \ldots, s_{k}$ and evaluated at $z_{1}, \ldots, z_{k}$. Also, we denote the vertical concatenation of the bilinear terms by $\tilde{\mathcal{N}}(s, \mu)=\left[\begin{array}{c}\mathcal{N}_{1}(s, \mu) \\ \vdots \\ \mathcal{N}_{m}(s, \mu)\end{array}\right]$.

\section{Structured interpolation}

Interpolatory model reduction has been one of the most commonly used and effective approaches to model reduction and shown to provide locally optimal reduced models for linear, bilinear, quadratic-bilinear dynamical systems; we refer the reader to $[3,6,19]$ and references therein for details on interpolatory model reduction for linear and nonlinear systems. In this setting, one chooses $V$ and $W$ in (7) such that the reduced-order transfer functions interpolate the transfer functions of the original system at selected points. In the setting of parametric structured multivariate transfer functions $G_{k}$ in (5), we want to construct $V$ and $W$ such that the reduced transfer functions $\widehat{G}_{k}$ in (6) satisfy

$$
\begin{aligned}
G_{k}\left(\sigma_{1}, \ldots, \sigma_{k}, \hat{\mu}\right) & =\widehat{G}_{k}\left(\sigma_{1}, \ldots, \sigma_{k}, \hat{\mu}\right) \text { and } \\
\nabla G_{k}\left(\sigma_{1}, \ldots, \sigma_{k}, \hat{\mu}\right) & =\nabla \widehat{G}_{k}\left(\sigma_{1}, \ldots, \sigma_{k}, \hat{\mu}\right)
\end{aligned}
$$

for given frequency interpolation points $\sigma_{1}, \ldots, \sigma_{k} \in \mathbb{C}$ and the parameter interpolation point $\mu \in \mathbb{M}$ where $\nabla G_{k}$ denotes the Jacobian matrix

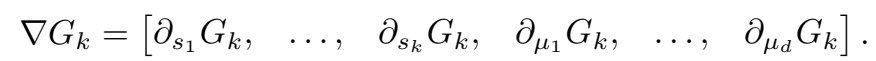

We emphasize that for multi-input/multi-output (MIMO) systems we consider here, transfer functions $G_{k}$ are matrix valued. Therefore, conditions in (8) and (9) enforce matrix interpolation. This is not usually needed. For MIMO linear dynamical systems, for example, one enforces tangential interpolation, meaning matrixinterpolation along selected directions [3]. However, for brevity and to keep the notation concise, we will focus on matrix interpolation.

Even though we have only listed two sets of interpolation conditions in (8) and (9), Theorems 1 and 2 below will show how to construct $V$ and $W$ to enforce interpolation for more general cases, including higher-order partial derivatives. The recent work [8] showed how to enforce (8) and (9) for non-parametric structured bilinear systems. Our theory below will extend these results to the parametric case. Note that the first condition (8) does not involve any differentiation with respect to the parameter $\hat{\mu}$ and can be viewed as interpolation for a fixed parameter $\mu=\hat{\mu}$. Therefore, we might expect that the subspace constructions from [8] for the non-parametric problem might yield the desired subspaces. This is indeed what we discuss first in Theorems 1 and 2. However, the second condition (9) involves matching sensitivity with respect to the parameter as well, which will be discussed in Section 4.

Theorem 1 (Structured matrix interpolation). Let $G$ be a parametric bilinear system, with its structured subsystem transfer functions $G_{k}$ in (5), and $\widehat{G}$ be the reducedorder parametric bilinear system, constructed as in (7) with its subsystem transfer functions $\widehat{G}_{k}$ in (6). Let the matrix functions $\mathcal{C}(s, \mu), \mathcal{K}(s, \mu)^{-1}, \mathcal{N}(s, \mu), \mathcal{B}(s, \mu)$, and $\widehat{\mathcal{K}}(s, \mu)^{-1}$ be defined for given sets of frequency interpolation points $\sigma_{1}, \ldots, \sigma_{k} \in \mathbb{C}$ and $\varsigma_{1}, \ldots, \varsigma_{\theta} \in \mathbb{C}$, and the parameter interpolation point $\hat{\mu} \in \mathbb{M}$.

(a) If $V$ is constructed such that

$$
\operatorname{span}(V) \supseteq \operatorname{span}\left(\left[V_{1}, \ldots, V_{k}\right]\right),
$$

where

$$
\begin{aligned}
V_{1} & =\mathcal{K}\left(\sigma_{1}, \hat{\mu}\right)^{-1} \mathcal{B}\left(\sigma_{1}, \hat{\mu}\right) \text { and } \\
V_{j} & =\mathcal{K}\left(\sigma_{j}, \hat{\mu}\right)^{-1} \mathcal{N}\left(\sigma_{j-1}, \hat{\mu}\right)\left(I_{m} \otimes V_{j-1}\right),
\end{aligned}
$$

for $2 \leq j \leq k$, then the following interpolation conditions hold true:

$$
G_{j}\left(\sigma_{1}, \ldots, \sigma_{j}, \hat{\mu}\right)=\widehat{G}_{j}\left(\sigma_{1}, \ldots, \sigma_{j}, \hat{\mu}\right),
$$

for $j=1, \ldots, k$.

(b) If $W$ is constructed such that

$$
\operatorname{span}(W) \supseteq \operatorname{span}\left(\left[W_{1}, \ldots, W_{\theta}\right]\right),
$$

where

$$
\begin{aligned}
W_{1} & =\mathcal{K}\left(\varsigma_{\theta}, \hat{\mu}\right)^{-\mathrm{H}} \mathcal{C}\left(\varsigma_{\theta}, \hat{\mu}\right)^{\mathrm{H}} \text { and } \\
W_{i} & =\mathcal{K}\left(\varsigma_{\theta-i+1}, \hat{\mu}\right)^{-\mathrm{H}} \tilde{\mathcal{N}}\left(\varsigma_{\theta-i+1}, \hat{\mu}\right)^{\mathrm{H}}\left(I_{m} \otimes W_{i-1}\right),
\end{aligned}
$$


for $2 \leq i \leq \theta$, then the following interpolation conditions hold true:

$$
G_{i}\left(\varsigma_{\theta-i+1}, \ldots, \varsigma_{\theta}, \hat{\mu}\right)=\widehat{G}_{i}\left(\varsigma_{\theta-i+1}, \ldots, \varsigma_{\theta}, \hat{\mu}\right),
$$

for $i=1, \ldots, \theta$.

(c) Let $V$ be constructed as in Part (a) and $W$ as in Part (b). Then, in addition to (11) and (12), the interpolation conditions

$$
\begin{aligned}
& G_{q+\eta}\left(\sigma_{1}, \ldots, \sigma_{q}, \varsigma_{\theta-\eta+1}, \ldots, \varsigma_{\theta}, \hat{\mu}\right) \\
& =\widehat{G}_{q+\eta}\left(\sigma_{1}, \ldots, \sigma_{q}, \varsigma_{\theta-\eta+1}, \ldots, \varsigma_{\theta}, \hat{\mu}\right),
\end{aligned}
$$

hold for $1 \leq q \leq k$ and $1 \leq \eta \leq \theta$.

Proof. Given the fixed parameter $\hat{\mu} \in \mathbb{M}$, the matrix functions $\mathcal{C}(s, \hat{\mu}), \mathcal{K}(s, \hat{\mu}), \mathcal{N}(s, \hat{\mu})$ and $\mathcal{B}(s, \hat{\mu})$ can be viewed as the realization of a non-parametric bilinear system. Then, the interpolation conditions (11)-(13) can be considered as subsystem interpolation of a non-parametric bilinear system as these conditions do not involve any variation/sensitivity with respect to $\mu$. Therefore, the subspace conditions in [8, Theorem 8], for interpolating a non-parametric structured bilinear system, apply here as well, which are precisely the subspace conditions listed in Parts (a)-(c). However, to make the paper self-contained and the proof of Theorem 3 in Section 4 easier to follow, we will still prove Part (a) for $k=2$. By induction over $k$, the rest of the result in (a) follows directly using the same arguments. Using (6), the second reduced-order transfer function is given by

$$
\begin{aligned}
\widehat{G}_{2}\left(\sigma_{1}, \sigma_{2}, \hat{\mu}\right)= & \widehat{\mathcal{C}}\left(\sigma_{2}, \hat{\mu}\right) \widehat{\mathcal{K}}\left(\sigma_{2}, \hat{\mu}\right)^{-1} \widehat{\mathcal{N}}\left(\sigma_{1}, \hat{\mu}\right) \\
& \times\left(I_{m} \otimes \widehat{\mathcal{K}}\left(\sigma_{1}, \hat{\mu}\right)^{-1}\right)\left(I_{m} \otimes \widehat{\mathcal{B}}\left(\sigma_{1}, \hat{\mu}\right)\right) .
\end{aligned}
$$

We observe that with (7) it holds

$$
\begin{aligned}
& \left(I_{m} \otimes V\right)\left(I_{m} \otimes \widehat{\mathcal{K}}\left(\sigma_{1}, \hat{\mu}\right)^{-1}\right)\left(I_{m} \otimes \widehat{\mathcal{B}}\left(\sigma_{1}, \hat{\mu}\right)\right) \\
& =\left(I_{m} \otimes V \widehat{\mathcal{K}}\left(\sigma_{1}, \hat{\mu}\right)^{-1} \widehat{\mathcal{B}}\left(\sigma_{1}, \hat{\mu}\right)\right) \\
& =\left(I_{m} \otimes V \widehat{\mathcal{K}}\left(\sigma_{1}, \hat{\mu}\right)^{-1} W^{\mathrm{H}} \mathcal{B}\left(\sigma_{1}, \hat{\mu}\right)\right) \\
& =(I_{m} \otimes \underbrace{V \widehat{\mathcal{K}}\left(\sigma_{1}, \hat{\mu}\right)^{-1} W^{\mathrm{H}} \mathcal{K}\left(\sigma_{1}, \hat{\mu}\right)}_{P_{\mathrm{V}_{1}}} \underbrace{\mathcal{K}\left(\sigma_{1}, \hat{\mu}\right)^{-1} \mathcal{B}\left(\sigma_{1}, \hat{\mu}\right)}_{V_{1}}),
\end{aligned}
$$

where $P_{\mathrm{V}_{1}}$ is a projector onto $\operatorname{span}(V)$ and $V_{1}$ is as defined in (10). By construction, we have $\operatorname{span}\left(V_{1}\right) \subseteq \operatorname{span}(V)$; thus $P_{\mathrm{V}_{1}} V_{1}=V_{1}$ and, therefore

$$
\begin{aligned}
& \left(I_{m} \otimes V\right)\left(I_{m} \otimes \widehat{\mathcal{K}}\left(\sigma_{1}, \hat{\mu}\right)^{-1}\right)\left(I_{m} \otimes \widehat{\mathcal{B}}\left(\sigma_{1}, \hat{\mu}\right)\right) \\
& =\left(I_{m} \otimes \mathcal{K}\left(\sigma_{1}, \hat{\mu}\right)^{-1}\right)\left(I_{m} \otimes \mathcal{B}\left(\sigma_{1}, \hat{\mu}\right)\right) .
\end{aligned}
$$

Then, $\widehat{G}_{2}$ can be written as

$$
\begin{aligned}
\widehat{G}_{2}\left(\sigma_{1}, \sigma_{2}, \hat{\mu}\right)= & \widehat{\mathcal{C}}\left(\sigma_{2}, \hat{\mu}\right) \widehat{\mathcal{K}}\left(\sigma_{2}, \hat{\mu}\right)^{-1} W^{\mathrm{H}} \mathcal{N}\left(\sigma_{1}, \hat{\mu}\right) \\
& \times\left(I_{m} \otimes \mathcal{K}\left(\sigma_{1}, \hat{\mu}\right)^{-1}\right)\left(I_{m} \otimes \mathcal{B}\left(\sigma_{1}, \hat{\mu}\right)\right)
\end{aligned}
$$

$$
\begin{aligned}
= & \mathcal{C}\left(\sigma_{2}, \hat{\mu}\right) V \widehat{\mathcal{K}}\left(\sigma_{2}, \hat{\mu}\right)^{-1} W^{\mathrm{H}} \mathcal{N}\left(\sigma_{1}, \hat{\mu}\right) \\
& \times\left(I_{m} \otimes V_{1}\right) .
\end{aligned}
$$

Also, it holds that

$$
\begin{aligned}
& V \widehat{\mathcal{K}}\left(\sigma_{2}, \hat{\mu}\right)^{-1} W^{\mathrm{H}} \mathcal{N}\left(\sigma_{1}, \hat{\mu}\right)\left(I_{m} \otimes V_{1}\right) \\
& =\underbrace{V \widehat{\mathcal{K}}\left(\sigma_{2}, \hat{\mu}\right)^{-1} W^{\mathrm{H}} \mathcal{K}\left(\sigma_{2}, \hat{\mu}\right)}_{P_{\mathrm{V}_{2}}} \\
& \quad \times \underbrace{\mathcal{K}\left(\sigma_{2}, \hat{\mu}\right)^{-1} \mathcal{N}\left(\sigma_{1}, \hat{\mu}\right)\left(I_{m} \otimes V_{1}\right)}_{V_{2}} \\
& =\mathcal{K}\left(\sigma_{2}, \hat{\mu}\right)^{-1} \mathcal{N}\left(\sigma_{1}, \hat{\mu}\right)\left(I_{m} \otimes V_{1}\right),
\end{aligned}
$$

using the fact that $P_{\mathrm{V}_{2}}$ is another projector onto $\operatorname{span}(V)$ and that $\operatorname{span}\left(V_{2}\right) \subseteq \operatorname{span}(V)$. Inserting this last equality into the second reduced-order transfer function yields

$$
\widehat{G}_{2}\left(\sigma_{1}, \sigma_{2}, \hat{\mu}\right)=G_{2}\left(\sigma_{1}, \sigma_{2}, \hat{\mu}\right) .
$$

Constructing further projectors onto $\operatorname{span}(V)$ for higherorder transfer functions gives the result in (a). The result in Part (b) follows exactly the same way by using the Hermitian transposed matrix functions and constructing now projectors onto $\operatorname{span}(W)$. Part (c) is then resulting from the application of both types of projectors onto $\operatorname{span}(V)$ and $\operatorname{span}(W)$.

In Theorem 1, only function values are matched, i.e., the zeroth derivative. The following theorem extends these results to matching higher-order derivatives in the frequency arguments, i.e., to enforcing Hermite interpolation conditions.

Theorem 2 (Hermite matrix interpolation). Let $G$ be a parametric bilinear system, with its structured subsystem transfer functions $G_{k}$ in (5) and $\widehat{G}$ be the reducedorder parametric bilinear system, constructed as in (7) with its subsystem transfer functions $\widehat{G}_{k}$ in (6). Let the matrix functions $\mathcal{C}(s, \mu), \mathcal{K}(s, \mu)^{-1}, \mathcal{N}(s, \mu), \mathcal{B}(s, \mu)$, and $\widehat{\mathcal{K}}(s, \mu)^{-1}$ be analytic for given sets of frequency interpolation points $\sigma_{1}, \ldots, \sigma_{k} \in \mathbb{C}$ and $\varsigma_{1}, \ldots, \varsigma_{\theta} \in \mathbb{C}$, and the parameter interpolation point $\hat{\mu} \in \mathbb{M}$.

(a) If $V$ is constructed such that

$$
\operatorname{span}(V) \supseteq \operatorname{span}\left(\left[V_{1,0}, \ldots, V_{k, \ell_{k}}\right]\right),
$$

where

$$
\begin{aligned}
V_{1, j_{1}}= & \partial_{s^{j_{1}}}\left(\mathcal{K}^{-1} \mathcal{B}\right)\left(\sigma_{1}, \hat{\mu}\right) \text { and } \\
V_{q, j_{q}}= & \partial_{s^{j}} \mathcal{K}^{-1}\left(\sigma_{q}, \hat{\mu}\right) \\
& \times\left(\prod _ { j = 1 } ^ { q - 2 } \partial _ { s ^ { \ell } { } ^ { - j } } \left(\left(I_{m^{j-1}} \otimes \mathcal{N}\right)\right.\right. \\
& \left.\left.\times\left(I_{m^{j}} \otimes \mathcal{K}\right)\right)\left(\sigma_{q-j}, \hat{\mu}\right)\right) \\
& \times \partial_{s^{\ell_{1}}}\left(\left(I_{m^{q-2}} \otimes \mathcal{N}\right)\left(I_{m^{q-1}} \otimes \mathcal{K}\right)\right. \\
& \left.\times\left(I_{m^{q-1}} \otimes \mathcal{B}\right)\right)\left(\sigma_{1}, \hat{\mu}\right),
\end{aligned}
$$


for $2 \leq q \leq k$ and $0 \leq j_{1} \leq \ell_{1} ; 0 \leq j_{q} \leq \ell_{q}$, then the following interpolation conditions hold true:

$$
\begin{aligned}
& \partial_{s_{1}^{\ell_{1}} \ldots s_{q-1}^{\ell_{q-1}} s_{q}^{j_{q}}} G_{q}\left(\sigma_{1}, \ldots, \sigma_{q}, \hat{\mu}\right) \\
& =\partial_{s_{1}^{\ell_{1}} \ldots s_{q-1}^{\ell_{q-1}} s_{q}^{j_{q}}} \widehat{G}_{q}\left(\sigma_{1}, \ldots, \sigma_{q}, \hat{\mu}\right),
\end{aligned}
$$

for $q=1, \ldots, k$ and $j_{q}=0, \ldots, \ell_{q}$.

(b) If $W$ is constructed such that

$$
\operatorname{span}(W) \supseteq \operatorname{span}\left(\left[W_{1,0}, \ldots, W_{\theta, \nu_{\theta}}\right]\right),
$$

where

$$
\begin{aligned}
W_{1, i_{\theta}}= & \partial_{s^{i \theta}}\left(\mathcal{K}^{-\mathrm{H}} \mathcal{C}^{\mathrm{H}}\right)\left(\varsigma_{\theta}, \hat{\mu}\right) \text { and } \\
W_{\eta, i_{\theta-\eta+1}}= & \partial_{s^{i} \theta-\eta+1}\left(\mathcal{K}^{-\mathrm{H}} \tilde{\mathcal{N}}^{\mathrm{H}}\right)\left(\varsigma_{\theta-\eta+1}, \hat{\mu}\right) \\
& \times\left(\prod _ { i = \theta - \eta + 2 } ^ { \theta - 1 } \partial _ { s ^ { \nu _ { i } } } \left(I_{m^{i-1}} \otimes \mathcal{K}^{-\mathrm{H}}\right.\right. \\
& \left.\left.\times \tilde{\mathcal{N}}^{\mathrm{H}}\right)\left(\varsigma_{i}, \hat{\mu}\right)\right) \\
& \times\left(I_{m^{\theta-1}} \otimes \partial_{s^{\nu_{\theta}}}\left(\mathcal{K}^{-\mathrm{H}} \mathcal{C}^{\mathrm{H}}\right)\left(\varsigma_{\theta}, \hat{\mu}\right)\right),
\end{aligned}
$$

for $2 \leq \eta \leq \theta$ and $0 \leq i_{\theta} \leq \nu_{\theta} ; 0 \leq i_{\theta-\eta+1} \leq \nu_{\theta-\eta+1}$, then the following interpolation conditions hold true:

$$
\begin{aligned}
& \partial_{s_{1} i_{\theta-\eta+1}} s_{2}^{\nu_{2} \ldots s_{\theta}^{\nu}} G_{\eta}\left(\varsigma_{\theta-\eta+1}, \ldots, \varsigma_{\theta}, \hat{\mu}\right) \\
& =\partial_{s_{1}^{i_{\theta}-\eta+1}} s_{2}^{\nu_{2}} \cdots s_{\theta}^{\nu_{\theta}} \widehat{G}_{\eta}\left(\varsigma_{\theta-\eta+1}, \ldots, \varsigma_{\theta}, \hat{\mu}\right),
\end{aligned}
$$

for $\eta=1, \ldots, \theta$ and $i_{\eta}=0, \ldots, \nu_{\eta}$.

(c) Let $V$ be constructed as in (a) and $W$ as in (b). Then, in addition to (14) and (15), the interpolation conditions (16) hold for $j_{q}=0, \ldots, \ell_{q} ; i_{\theta-\eta+1}=0$, $\ldots, \nu_{\theta-\eta+1} ; 1 \leq q \leq k$ and $1 \leq \eta \leq \theta$.

Proof. As in Theorem 1, all the interpolation conditions are for a fixed parameter $\hat{\mu} \in \mathbb{M}$, i.e., they can be proven using a similar construction of projectors onto suitable subspaces as in Theorem 1. Therefore, the subspace conditions in [8, Theorem 9] can be applied here, which are precisely the subspace conditions listed in Theorem 2.

\section{Matching parameter sensitivities}

So far, the interpolation conditions enforced did not show variability with respect to the parameter $\mu$. Even in the Hermite conditions matched in Theorem 2, the matched derivatives (sensitivities) are with respect to the frequency points. This enabled us to directly employ the conditions and analysis from [8]. However, for parametric systems it is important to match the parameter sensitivity with respect to the parameter variation as well.
This is what we establish in the next result, extending the similar results from linear dynamics [5] and unstructured bilinear dynamics [16] to the new parametric structured framework. An important conclusion is that the parameter sensitivity is matched implicitly, i.e., without ever explicitly computing it. This is achieved by using the same set of frequency interpolation points for $V$ and $W$.

Theorem 3 (Two-sided matrix interpolation with identical point sets). Let $G$ be a parametric bilinear system, with its structured subsystem transfer functions $G_{k}$ in (5) and $\widehat{G}$ be the reduced-order parametric bilinear system, constructed as in (7) with its subsystem transfer functions $\widehat{G}_{k}$ in (6). Let the matrix functions $\mathcal{C}(s, \mu), \mathcal{K}(s, \mu)^{-1}$, $\mathcal{N}(s, \mu), \mathcal{B}(s, \mu)$, and $\widehat{\mathcal{K}}(s, \mu)^{-1}$ be analytic for a given set of frequency interpolation points $\sigma_{1}, \ldots, \sigma_{k} \in \mathbb{C}$ and the parameter interpolation point $\hat{\mu} \in \mathbb{M}$.

(a) Let $V$ be constructed as in Theorem 1 Part (a) and $W$ be constructed as in Theorem 1 Part (b) with $\varsigma_{i}=\sigma_{i}$ for $i=1,2, \ldots, k$. Then, in addition to (11)-(13) it holds

$$
\nabla G_{k}\left(\sigma_{1}, \ldots, \sigma_{k}, \hat{\mu}\right)=\nabla \widehat{G}_{k}\left(\sigma_{1}, \ldots, \sigma_{k}, \hat{\mu}\right) .
$$

(b) Let $V$ be constructed as in Theorem 2 Part (a) and $W$ be constructed as in Theorem 2 Part (b) with $\varsigma_{i}=\sigma_{i}$ for $i=1,2, \ldots, k$. Then, in addition to (14)-(16), it holds

$$
\begin{aligned}
& \nabla\left(\partial_{s_{1}^{\ell_{1} \ldots s_{k} \ell_{k}}} G_{k}\left(\sigma_{1}, \ldots, \sigma_{k}, \hat{\mu}\right)\right) \\
& =\nabla\left(\partial_{s_{1}^{\ell_{1} \ldots s_{k}}} \widehat{G}_{k}\left(\sigma_{1}, \ldots, \sigma_{k}, \hat{\mu}\right)\right) .
\end{aligned}
$$

Proof. For brevity, we only prove (17). The proof of (18) follows analogously. As in the proof of Theorem 1, we will construct appropriate projectors onto the projection spaces $\operatorname{span}(V)$ or $\operatorname{span}(W)$. In contrast to Theorem 2, we now also interpolate the derivative with respect to the parameters. Using the product rule, the partial derivative of $\widehat{G}_{k}$ with respect to a single parameter entry $\mu_{i}$, for $1 \leq i \leq d$, is given by

$$
\begin{aligned}
& \partial_{\mu_{i}} \widehat{G}_{k}\left(\sigma_{1}, \ldots, \sigma_{k}, \hat{\mu}\right) \\
& =\sum_{\alpha \in \mathbb{A}}\left(\partial_{\mu_{i}^{\alpha_{1}}} \widehat{\mathcal{C}}\left(\sigma_{k}, \hat{\mu}\right)\right)\left(\partial_{\mu_{i}^{\alpha_{2}}} \widehat{\mathcal{K}}^{-1}\left(\sigma_{k}, \hat{\mu}\right)\right) \\
& \quad \times\left(\prod_{j=1}^{k-1}\left(I_{m^{j-1}} \otimes \partial_{\mu_{i}^{\alpha_{2 j+1}}} \widehat{\mathcal{N}}\left(\sigma_{k-j}, \hat{\mu}\right)\right)\right. \\
& \left.\quad \times\left(I_{m^{j}} \otimes \partial_{\mu_{i}^{\alpha_{2 j+2}}} \widehat{\mathcal{K}}^{-1}\left(\sigma_{k-j}, \hat{\mu}\right)\right)\right) \\
& \quad \times\left(I_{m^{k-1}} \otimes \partial_{\mu_{i}^{\alpha_{2 k+1}}} \widehat{\mathcal{B}}\left(\sigma_{1}, \hat{\mu}\right)\right),
\end{aligned}
$$

where $\mathbb{A}$ denotes the set of all columns of the identity matrix of size $2 k+1$. In other words, (19) is a sum of $2 k+1$ 


$$
\begin{aligned}
& \partial_{s_{1}^{\ell_{1}} \ldots s_{q-1}^{\ell} s_{q-1}^{j_{q}} s_{q+1}^{i_{\theta-\eta+1}} s_{q+2}^{\nu_{\theta-\eta+2}} \ldots s_{q+\eta}^{\nu_{\theta}}} G_{q+\eta}\left(\sigma_{1}, \ldots, \sigma_{q}, \varsigma_{\theta-\eta+1}, \ldots, \varsigma_{\theta}, \hat{\mu}\right) \\
& =\partial_{s_{1}^{\ell_{1}} \ldots s_{q-1}^{\ell_{q-1}} s_{q}^{j_{q}} s_{q+1}^{i_{\theta-\eta+1}} s_{q+2}^{\nu_{\theta-\eta+2}} \ldots s_{q+\eta}^{\nu_{\theta}}} \widehat{G}_{q+\eta}\left(\sigma_{1}, \ldots, \sigma_{q}, \varsigma_{\theta-\eta+1}, \ldots, \varsigma_{\theta}, \hat{\mu}\right),
\end{aligned}
$$

terms where each term corresponds to the vector $\alpha$ taking a value from this set of columns. Therefore, in each term only a single matrix function is differentiated. We will show that every single term in the sum (19) matches the same term in the full order model, thus, summed together, proving the desired interpolation property (17). Consider, e.g., the second term in (19), i.e., the term in which $\alpha$ is the second column of the identity matrix: $\alpha=\left[\begin{array}{lllll}\alpha_{1} & \alpha_{2} & \alpha_{3} & \cdots & \alpha_{2 k+1}\end{array}\right]^{\top}=\left[\begin{array}{lllll}0 & 1 & 0 & \ldots & 0\end{array}\right]^{\top}$. Denote the corresponding term by $\widehat{\mathcal{H}}_{2}$. Then,

$$
\begin{aligned}
\widehat{\mathcal{H}}_{2}:= & \widehat{\mathcal{C}}\left(\sigma_{k}, \hat{\mu}\right)\left(\partial_{\mu_{i}} \widehat{\mathcal{K}}^{-1}\left(\sigma_{k}, \hat{\mu}\right)\right) \\
& \times\left(\prod_{j=1}^{k-1}\left(I_{m^{j-1}} \otimes \widehat{\mathcal{N}}\left(\sigma_{k-j}, \hat{\mu}\right)\right)\right. \\
& \left.\times\left(I_{m^{j}} \otimes \widehat{\mathcal{K}}\left(\sigma_{k-j}, \hat{\mu}\right)^{-1}\right)\right) \\
& \times\left(I_{m^{k-1}} \otimes \widehat{\mathcal{B}}\left(\sigma_{1}, \hat{\mu}\right)\right) .
\end{aligned}
$$

The derivative of the inverse appearing in $\widehat{\mathcal{H}}_{2}$ is given by $\partial_{\mu_{i}} \widehat{\mathcal{K}}^{-1}\left(\sigma_{k}, \hat{\mu}\right)=-\widehat{\mathcal{K}}\left(\sigma_{k}, \hat{\mu}\right)^{-1}\left(\partial_{\mu_{i}} \widehat{\mathcal{K}}\left(\sigma_{k}, \hat{\mu}\right)\right) \widehat{\mathcal{K}}\left(\sigma_{k}, \hat{\mu}\right)^{-1}$.

Therefore, $\widehat{\mathcal{H}}_{2}$ can be rewritten as

$$
\begin{aligned}
\widehat{\mathcal{H}}_{2}= & -\widehat{\mathcal{C}}\left(\sigma_{k}, \hat{\mu}\right) \widehat{\mathcal{K}}\left(\sigma_{k}, \hat{\mu}\right)^{-1}\left(\partial_{\mu_{i}} \widehat{\mathcal{K}}\left(\sigma_{k}, \hat{\mu}\right)\right) \widehat{\mathcal{K}}\left(\sigma_{k}, \hat{\mu}\right)^{-1} \\
& \times\left(\prod_{j=1}^{k-1}\left(I_{m^{j-1}} \otimes \widehat{\mathcal{N}}\left(\sigma_{k-j}, \hat{\mu}\right)\right)\right. \\
& \left.\times\left(I_{m^{j}} \otimes \widehat{\mathcal{K}}\left(\sigma_{k-j}, \hat{\mu}\right)^{-1}\right)\right) \\
& \times\left(I_{m^{k-1}} \otimes \widehat{\mathcal{B}}\left(\sigma_{1}, \hat{\mu}\right)\right) \\
= & :-\widehat{W}_{1}^{\mathrm{H}}\left(\partial_{\mu_{i}} \widehat{\mathcal{K}}\left(\sigma_{k}, \hat{\mu}\right)\right) \widehat{V}_{k} .
\end{aligned}
$$

Noting that the model reduction space $V$ were constructed as in Theorem 1, we obtain

$$
\begin{aligned}
V \widehat{V}_{k}= & V \widehat{\mathcal{K}}\left(\sigma_{k}, \hat{\mu}\right)^{-1}\left(\prod_{j=1}^{k-1}\left(I_{m^{j-1}} \otimes \widehat{\mathcal{N}}\left(\sigma_{k-j}, \hat{\mu}\right)\right)\right. \\
& \left.\times\left(I_{m^{j}} \otimes \widehat{\mathcal{K}}\left(\sigma_{k-j}, \hat{\mu}\right)^{-1}\right)\right) \\
& \times\left(I_{m^{k-1}} \otimes \widehat{\mathcal{B}}\left(\sigma_{1}, \hat{\mu}\right)\right)
\end{aligned}
$$

$$
\begin{aligned}
= & \underbrace{V \widehat{\mathcal{K}}\left(\sigma_{k}, \hat{\mu}\right)^{-1} W^{\mathrm{H}} \mathcal{K}\left(\sigma_{k}, \hat{\mu}\right)}_{P_{\mathrm{V}_{k}}} \\
& \times \mathcal{K}\left(\sigma_{k}, \hat{\mu}\right)^{-1}\left(\prod_{j=1}^{k-1}\left(I_{m^{j-1}} \otimes \mathcal{N}\left(\sigma_{k-j}, \hat{\mu}\right)\right)\right. \\
& \left.\times\left(I_{m^{j}} \otimes \mathcal{K}\left(\sigma_{k-j}, \hat{\mu}\right)^{-1}\right)\right) \\
& \times\left(I_{m^{k-1}} \otimes \mathcal{B}\left(\sigma_{1}, \hat{\mu}\right)\right) \\
= & P_{\mathrm{V}_{k}} V_{k} \\
= & V_{k},
\end{aligned}
$$

where $P_{\mathrm{V}_{k}}$ is a projector onto $\operatorname{span}(V)$. Similarly, we have

$$
\begin{aligned}
W \widehat{W}_{1} & =W \widehat{\mathcal{K}}\left(\sigma_{k}, \hat{\mu}\right)^{-\mathrm{H}} \widehat{\mathcal{C}}\left(\sigma_{k}, \hat{\mu}\right)^{\mathrm{H}} \\
& =\underbrace{W \widehat{\mathcal{K}}\left(\sigma_{k}, \hat{\mu}\right)^{-\mathrm{H}} V \mathcal{K}\left(\sigma_{k}, \hat{\mu}\right)^{\mathrm{H}}}_{P_{\mathrm{W}_{1}}} \underbrace{\mathcal{K}\left(\sigma_{k}, \hat{\mu}\right)^{-\mathrm{H}} \mathcal{C}\left(\sigma_{k}, \hat{\mu}\right)^{\mathrm{H}}}_{W_{1}} \\
& =W_{1},
\end{aligned}
$$

with $P_{\mathrm{W}_{1}}$ a projector onto $\operatorname{span}(W)$. Using those two identities, we obtain

$$
\begin{aligned}
\widehat{\mathcal{H}}_{2}= & -\widehat{W}_{1}^{\mathrm{H}}\left(\partial_{\mu_{i}} \widehat{\mathcal{K}}\left(\sigma_{k}, \hat{\mu}\right)\right) \widehat{V}_{k} \\
= & -\widehat{W}_{1}^{\mathrm{H}} W^{\mathrm{H}}\left(\partial_{\mu_{i}} \mathcal{K}\left(\sigma_{k}, \hat{\mu}\right)\right) V \widehat{V}_{k} \\
= & -W_{1}^{\mathrm{H}}\left(\partial_{\mu_{i}} \mathcal{K}\left(\sigma_{k}, \hat{\mu}\right)\right) V_{k} \\
= & \mathcal{C}\left(\sigma_{k}, \hat{\mu}\right)\left(\partial_{\mu_{i}} \mathcal{K}^{-1}\left(\sigma_{k}, \hat{\mu}\right)\right) \\
& \times\left(\prod_{j=1}^{k-1}\left(I_{m^{j-1}} \otimes \mathcal{N}\left(\sigma_{k-j}, \hat{\mu}\right)\right)\right. \\
& \left.\times\left(I_{m^{j}} \otimes \mathcal{K}\left(\sigma_{k-j}, \hat{\mu}\right)^{-1}\right)\right) \\
& \times\left(I_{m^{k-1}} \otimes \mathcal{B}\left(\sigma_{1}, \hat{\mu}\right)\right),
\end{aligned}
$$

i.e., $\widehat{\mathcal{H}}_{2}$ is identical to the term using the original matrix functions. Since the same technique can be used for all other $\alpha$ values corresponding the other columns in the set $\mathbb{A}$, we obtain, for all $1 \leq i \leq d$,

$$
\partial_{\mu_{i}} \widehat{G}_{k}\left(\sigma_{1}, \ldots, \sigma_{k}, \hat{\mu}\right)=\partial_{\mu_{i}} G_{k}\left(\sigma_{1}, \ldots, \sigma_{k}, \hat{\mu}\right) .
$$

Interpolation of the partial derivatives with respect to the frequency parameters follows by using the fixed parameter $\hat{\mu}$ in [8, Corollary 2]. Together with (20), this proves (17). 


$$
\left.G_{k}\left(s_{1}, \ldots, s_{k}, \mu\right)=\mathcal{C}\left(s_{k}, \mu\right) \mathcal{K}\left(s_{k}, \mu\right)^{-1}\left(\prod_{j=1}^{k-1} \mathcal{N}\left(s_{k-j}, \mu\right) \mathcal{K}\left(s_{k-j}, \mu\right)^{-1}\right)\right) \mathcal{B}\left(s_{1}, \mu\right)
$$

Remark 1. Theorem 3 shows how to match the parameter sensitivity implicitly without ever computing this quantity. Matching the parameter sensitivities is important, especially in the setting of optimization and design. These results can be extended to match the parameter Hessian as well; compare to [16]. However, we skip those details for brevity.

Remark 2. All the results in Theorems 1 to 3 are formulated for a single parameter interpolation point $\hat{\mu} \in \mathbb{M}$. However, the results directly extend to interpolation at multiple parameter sampling points $\hat{\mu}^{(1)}, \ldots, \hat{\mu}^{(q)} \in \mathbb{M}$ by constructing the projection spaces for every parameter sample and then concatenating the resulting spaces into a single global projection space. As example, consider the task of interpolating

$$
\begin{aligned}
& G_{1}\left(\sigma_{1}, \hat{\mu}^{(1)}\right), \quad G_{2}\left(\sigma_{1}, \sigma_{2}, \hat{\mu}^{(1)}\right), \\
& G_{1}\left(\sigma_{3}, \hat{\mu}^{(2)}\right), \quad G_{2}\left(\sigma_{3},, \sigma_{4}, \hat{\mu}^{(2)}\right),
\end{aligned}
$$

with the four frequency points $\sigma_{1}, \sigma_{2}, \sigma_{3}, \sigma_{4}$ and the two parameter points $\hat{\mu}^{(1)}, \hat{\mu}^{(2)}$. Using Theorem 1 Part (a), we can construct basis matrices $V^{(1)}, V^{(2)}$ for the interpolation in either $\hat{\mu}^{(1)}$ or $\hat{\mu}^{(2)}$, respectively. The construction of a reduced-order model that satisfies all interpolation conditions (22) is then given by constructing $V$ such that

$$
\operatorname{span}(V) \supseteq \operatorname{span}\left(\left[V^{(1)}, V^{(2)}\right]\right) .
$$

Remark 3. The results simplify drastically for singleinput single-output (SISO) systems. In that case, the multivariate transfer functions corresponding to bilinear systems (5) can be written without Kronecker products (21) and the construction of the corresponding projection spaces simplifies such that no Kronecker products are involved anymore.

\section{Numerical examples}

We illustrate the analysis with two benchmark examples. The experiments reported here have been executed on a machine with $2 \operatorname{Intel}(\mathrm{R}) \operatorname{Xeon}(\mathrm{R})$ Silver 4110 $\mathrm{CPU}$ processors running at $2.10 \mathrm{GHz}$ and equipped with 192 GB total main memory. The computer is run on CentOS Linux release 7.5.1804 (Core) with MATLAB 9.7.0.1190202 (R2019b).

\subsection{Parametric bilinear time-delay system}

In the first example from [13], we consider a time-delayed heated rod modeled by a one-dimensional heat equation

$$
\partial_{t} v(\zeta, t)=\partial_{\zeta}^{2} v(\zeta, t)+a_{1}(\zeta) v(\zeta, t)+a_{2}(\zeta) v(\zeta, t-1)+u(t),
$$

with homogeneous Dirichlet boundary conditions. We parameterize the diffusivity using the coefficients

$$
a_{1}=-\mu \sin (\zeta) \text { and } a_{2}=\mu \sin (\zeta), \text { for } \mu \in[1,10] .
$$

The non-parametric example in [13] is recovered for $\mu=$ 2. After a spatial discretization, we obtain a parametric bilinear system of the form

$$
\begin{aligned}
& \dot{x}(t)=\left(A_{0}-\mu A_{d}\right) x(t)+\mu A_{d} x(t-1)+N x(t) u(t)+B u(t), \\
& y(t)=C x(t),
\end{aligned}
$$

with $m=p=1$ and $n=5000$. In our structured parametric setting, this model corresponds to the matrix functions

$$
\begin{aligned}
& \mathcal{K}(s, \mu)=s I_{n}-\left(A_{0}-\mu A_{d}\right)-\mu e^{-s} A_{d}, \\
& \mathcal{B}(s, \mu)=B, \mathcal{N}(s, \mu)=N, \text { and } \mathcal{C}(s, \mu)=C .
\end{aligned}
$$

The reduced-order model is constructed via Theorem 3 Part (a) with the frequency sampling points $\left\{ \pm 10^{-4} \mathrm{i}\right.$, $\left.\pm 10^{4} \mathrm{i}\right\}$ and the parameter sampling points $\{1,5.5,10\}$ for the first two transfer functions. By construction, the reduced-order model has the same parametric time-delay structure as the original model and the state-space dimension $r=24$.

Figure 1a shows the relative time response error in the output, given by

$$
\operatorname{err}_{1, \mathrm{t}}(t, \mu):=\frac{|y(t ; \mu)-\hat{y}(t ; \mu)|}{|y(t ; \mu)|},
$$

for $t \in[0,10]$ and $\mu \in[1,10]$, using the same test input signal as in [13], namely, $u(t)=0.05(\cos (10 t)+\cos (5 t))$. The maximum error in the time and parameter domain is

$$
\max _{\mu \in[1,10]}\left(\max _{t \in[0,10]} \operatorname{err}_{1, \mathrm{t}}(t, \mu)\right) \approx 9.993 \cdot 10^{-6},
$$

illustrating a high-fidelity parametric reduced model over the full parameter domain. Figure $1 \mathrm{~b}$ depicts the relative error in the first transfer function over the parameter range, computed as

$$
\operatorname{err}_{1, \mathrm{f}}\left(\omega_{1}, \mu\right):=\frac{\left|G_{1}\left(\omega_{1} \mathrm{i}, \mu\right)-\widehat{G}_{1}\left(\omega_{1} \mathrm{i}, \mu\right)\right|}{\left|G_{1}\left(\omega_{1} \mathrm{i}, \mu\right)\right|},
$$




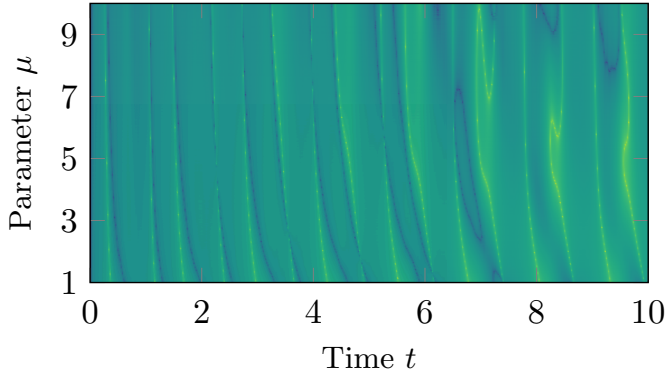

(a) Relative time domain error.

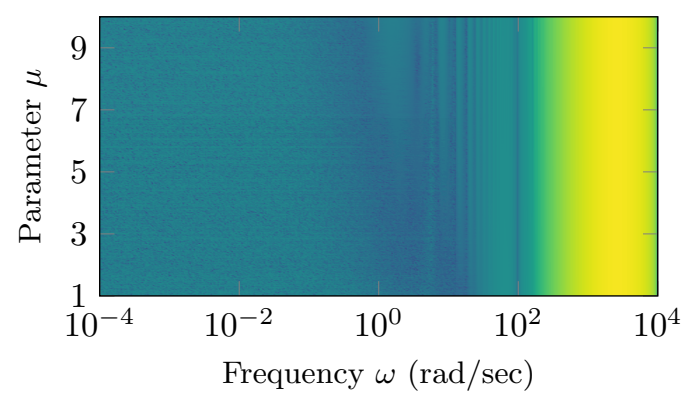

(b) Relative error in the first transfer function.

Figure 1: Relative errors for the time-delay system.

where $\omega_{1} \in\left[10^{-4}, 10^{4}\right]$ and $\mu \in[1,10]$. As for the time domain error, we computed the maximum error to obtain

$$
\max _{\mu \in[1,10]}\left(\max _{\omega_{1} \in\left[10^{-4}, 10^{4}\right]} \operatorname{err}_{1, \mathrm{f}}\left(\omega_{1}, \mu\right)\right) \approx 7.002 \cdot 10^{-6},
$$

showing the accuracy of the parametric reduced model in the frequency domain as well. We computed the maximum relative error in the second transfer function $G_{2}\left(s_{1}, s_{2}, \mu\right)$ as well to obtain

$$
\max _{\mu \in[1,10]}\left(\max _{\omega_{1}, \omega_{2} \in\left[10^{-4}, 10^{+4}\right]} \operatorname{err}_{1, \mathrm{f}}\left(\omega_{1}, \omega_{2}, \mu\right)\right) \approx 6.657 \cdot 10^{-4},
$$

where

$$
\operatorname{err}_{1, \mathrm{f}}\left(\omega_{1}, \omega_{2}, \mu\right):=\frac{\left|G_{2}\left(\omega_{1} \mathrm{i}, \omega_{2} \mathrm{i}, \mu\right)-\widehat{G}_{2}\left(\omega_{1} \mathrm{i}, \omega_{2} \mathrm{i}, \mu\right)\right|}{\left|G_{2}\left(\omega_{1} \mathrm{i}, \omega_{2} \mathrm{i}, \mu\right)\right|} .
$$

All these results show that the structure-preserving parametric reduced-order model is an accurate approximation of the original system over the full parameter domain.

\subsection{Parametric bilinear mechanical system}

As second example, we consider a parametrized version of the multi-input multi-output damped mass-spring system from [8], a special case of the model (1), given by

$$
\begin{aligned}
& M \ddot{x}(t ; \mu)+D \dot{x}(t ; \mu)+K x(t ; \mu)=B_{\mathrm{u}} u(t) \\
& \quad+\mu_{1} N_{\mathrm{p}, 1} x(t) u_{1}(t)+\mu_{2} N_{\mathrm{p}, 2} x(t) u_{2}(t), \\
& y(t ; \mu)=C_{\mathrm{p}} x(t ; \mu) \dot{x}(t ; \mu),
\end{aligned}
$$

where $\mu=\left(\mu_{1}, \mu_{2}\right)$ is the parameter entering through the bilinear terms and all the other matrices are exactly as in [8], except for $C_{\mathrm{p}}$, which, we set as $C_{\mathrm{p}}=\left[e_{2}, e_{n-3}\right]^{\top}$, where $e_{j}$ denotes the $j$-th column of the $n$-dimensional identity matrix. We have then $n=1000$ masses, $m=2$ inputs and $p=2$ outputs. The parameter set is $\mathbb{M}=$ $[0,1] \times[0,1]$. Note that for $\mu=(0,0)$, the system becomes linear. In our setting, this parametric bilinear model corresponds to

$$
\begin{aligned}
\mathcal{K}(s, \mu) & =s^{2} M+s D+K, \mathcal{B}(s, \mu)=B_{\mathrm{u}}, \\
\mathcal{N}(s, \mu) & =\left[\begin{array}{ll}
\mu_{1} N_{\mathrm{p}, 1} & \mu_{2} N_{\mathrm{p}, 2}
\end{array}\right], \text { and } \mathcal{C}(s, \mu)=C_{\mathrm{p}} .
\end{aligned}
$$

The reduced-order model is constructed via Theorem 1 with frequency interpolation points $\left\{ \pm 10^{-4} \mathrm{i}, \pm 10^{4} \mathrm{i}\right\}$ and the parameter interpolation points $\{(0,1),(1,0)\}$ for the first two transfer functions. To preserve the structural properties, such as positive definiteness of the mass matrix, of the single matrices, we use a one-sided projection, i.e., we choose $W=V$. Since the first transfer function (the linear term) is independent of the parameter, some of the vectors in the construction of $V$ are redundant and removed, yielding a structured parametric reduced-order model with $r=40$. We compute similar error quantities as in Section 5.1.

Figure 2a illustrates the relative time domain output error over the parameter range $\mu \in[0,1]^{2}$, computed as

$$
\operatorname{err}_{2, \mathrm{t}}(\mu):=\max _{j \in\{1,2\}}\left(\max _{t \in[0,100]} \frac{\left|y_{j}(. ; \mu)-\hat{y}_{j}(. ; \mu)\right|}{\left|y_{j}(. ; \mu)\right|}\right),
$$

using the input signal $u(t)=\left[\begin{array}{c}\sin (200 t)+200 \\ -\cos (200 t)-200\end{array}\right]$. The maximum error over the full parameter range is

$$
\max _{\mu \in[0,1]^{2}} \operatorname{err}_{2, \mathrm{t}}(\mu) \approx 8.849 \cdot 10^{-5}
$$

illustrating the high accuracy of the reduced model. Figure $2 \mathrm{~b}$ shows the relative error in the first transfer function approximation, i.e.,

$$
\operatorname{err}_{2, \mathrm{f}}\left(\omega_{1}\right):=\frac{\left\|G_{1}\left(\omega_{1} \mathrm{i}\right)-\widehat{G}_{1}\left(\omega_{1} \mathrm{i}\right)\right\|_{2}}{\|G(\omega \mathrm{i})\|_{2}},
$$

over the frequency range $\omega_{1} \in\left[10^{-4}, 10^{+4}\right]$, with the maximum attained error

$$
\max _{\omega_{1} \in\left[10^{-4}, 10^{+4}\right]} \operatorname{err}_{2, \mathrm{f}}\left(\omega_{1}\right) \approx 1.296 \cdot 10^{-4} .
$$

This error term is independent of the parameter since the first transfer function does not contain the parametric bilinear terms. We also computed the maximum relative approximation error for the second transfer function as $\max _{\mu \in[0,1]^{2}}\left(\max _{\omega_{1}, \omega_{2} \in\left[10^{-4}, 10^{+4}\right]} \operatorname{err}_{2, \mathrm{f}}\left(\omega_{1}, \omega_{1}, \mu\right)\right) \approx 1.496 \cdot 10^{-3}$, 


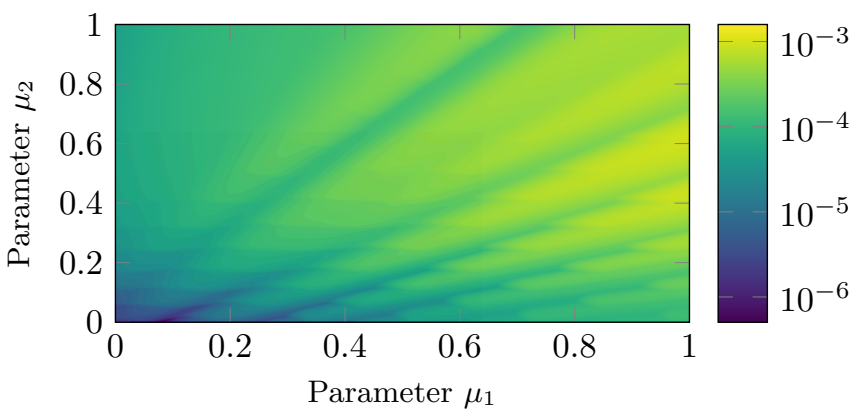

(a) Relative time domain error.

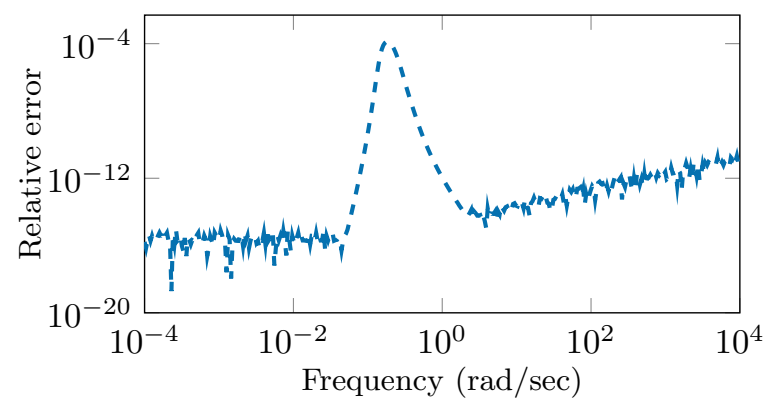

(b) Relative error in the first transfer function.

Figure 2: Relative errors for the damped mass-spring system.

where

$$
\operatorname{err}_{2, \mathrm{f}}\left(\omega_{1}, \omega_{1}, \mu\right):=\frac{\left\|G_{2}\left(\omega_{1} \mathrm{i}, \omega_{2} \mathrm{i}, \mu\right)-\widehat{G}_{2}\left(\omega_{1} \mathrm{i}, \omega_{2} \mathrm{i}, \mu\right)\right\|_{2}}{\left\|G_{2}\left(\omega_{1} \mathrm{i}, \omega_{2} \mathrm{i}, \mu\right)\right\|_{2}} .
$$

These numbers illustrate that the structured parametric approximation is a high-fidelity surrogate both in the frequency and time domains.

\section{Conclusions}

We have presented a structure-preserving interpolation framework for model order reduction of parametric bilinear systems. We have established the subspace conditions to enforce interpolation both in the frequency and parameter domains. Two numerical examples illustrate that the approach is well suited for efficient structurepreserving model order reduction of parametric bilinear systems. The presented approach covers arbitrary parameter dependencies of the system as well as more system structures than shown in the examples. An important open question is the appropriate choice of interpolation points in the frequency as well as the parameter domains to minimize the approximation error in some appropriate measure.

\section{Acknowledgment}

Benner and Werner were supported by the German Research Foundation (DFG) Research Training Group 2297 "MathCoRe", Magdeburg. Gugercin was supported in parts by National Science Foundation under Grant No. DMS-1720257 and DMS-1819110. Part of this material is based upon work supported by the National Science Foundation under Grant No. DMS-1439786 and by the Simons Foundation Grant No. 507536 while Gugercin and Benner were in residence at the Institute for Computational and Experimental Research in Mathematics in Providence, RI, during the "Model and dimension reduction in uncertain and dynamic systems" program.

\section{References}

[1] S. Al-Baiyat, A. S. Farag, and M. Bettayeb. Transient approximation of a bilinear two-area interconnected power system. Electric Power Systems Research, 26(1):11-19, 1993. doi:10.1016/ 0378-7796 (93) 90064-L.

[2] A. C. Antoulas, C. A. Beattie, and S. Gugercin. Interpolatory model reduction of large-scale dynamical systems. In Javad Mohammadpour and Karolos M. Grigoriadis, editors, Efficient Modeling and Control of Large-Scale Systems, pages 3-58. Springer US, 2010. doi:10.1007/978-1-4419-5757-3_1.

[3] A. C. Antoulas, C. A. Beattie, and S. Gugercin. Interpolatory Methods for Model Reduction. Computational Science \& Engineering. Society for Industrial and Applied Mathematics, Philadelphia, PA, 2020. doi:10.1137/1.9781611976083.

[4] Z. Bai and D. Skoogh. A projection method for model reduction of bilinear dynamical systems. Linear Algebra Appl., 415(2-3):406-425, 2006. doi: $10.1016 /$ j.laa.2005.04.032.

[5] U. Baur, C. A. Beattie, P. Benner, and S. Gugercin. Interpolatory projection methods for parameterized model reduction. SIAM J. Sci. Comput., 33(5):24892518, 2011. doi:10.1137/090776925.

[6] U. Baur, P. Benner, and L. Feng. Model order reduction for linear and nonlinear systems: A system-theoretic perspective. Arch. Comput. Methods Eng., 21(4):331-358, 2014. doi:10.1007/ s11831-014-9111-2.

[7] C. A. Beattie and S. Gugercin. Interpolatory projection methods for structure-preserving model reduction. Syst. Control Lett., 58(3):225-232, 2009. doi:10.1016/j.sysconle.2008.10.016. 
[8] P. Benner, S. Gugercin, and S. W. R. Werner. Structure-preserving interpolation of bilinear control systems. e-print 2005.00795, arXiv, 2020. math.NA. URL: https: //arxiv .org/abs/2005.00795.

[9] P. Benner, P. Kürschner, and J. Saak. An improved numerical method for balanced truncation for symmetric second order systems. Math. Comput. Model. Dyn. Syst., 19(6):593-615, 2013. doi: 10.1080/13873954.2013.794363.

[10] T. Breiten and T. Damm. Krylov subspace methods for model order reduction of bilinear control systems. Syst. Control Lett., 59(8):443-450, 2010. doi:10. $1016 / j$.sysconle.2010.06.003.

[11] M. Condon and R. Ivanov. Krylov subspaces from bilinear representations of nonlinear systems. Compel-Int. J. Comp. Math. Electr. Electron. Eng., 26(2):399-406, 2007. doi:10.1108/ 03321640710727755.

[12] L. Feng and P. Benner. A note on projection techniques for model order reduction of bilinear systems. In AIP Conference Proceedings, volume 936, pages 208-211, 2007. doi:10.1063/1.2790110.

[13] I. V. Gosea, I. Pontes Duff, P. Benner, and A. C. Antoulas. Model order reduction of bilinear timedelay systems. In 18th European Control Conference (ECC), pages 2289-2294, 2019. doi:10.23919/ECC. 2019.8796085 .

[14] R. R. Mohler. Bilinear Control Processes: With Applications to Engineering, Ecology and Medicine, volume 106 of Mathematics in Science and Engineering. Academic Press, New York, London, 1973.

[15] Y. Ou. Optimal Control of a Class of Nonlinear Parabolic PDE Systems Arising in Fusion Plasma Current Profile Dynamics. PhD thesis, Lehigh University, Bethlehem, Pennsylvania, USA, 2010.

[16] A. C. Rodriguez, S. Gugercin, and J. Boggaard. Interpolatory model reduction of parameterized bilinear dynamical systems. Adv. Comput. Math., 44(6):1887-1916, 2018. doi:10.1007/ s10444-018-9611-y.

[17] W. J. Rugh. Nonlinear System Theory: The Volterra/Wiener Approach. The Johns Hopkins University Press, Baltimore, 1981.

[18] J. Saputra, R. Saragih, and D. Handayani. Robust $H_{\infty}$ controller for bilinear system to minimize HIV concentration in blood plasma. J. Phys.: Conf. Ser., 1245:012055, 2019. doi :10.1088/1742-6596/1245/ $1 / 012055$.
[19] G. Scarciotti and A. Astolfi. Nonlinear model reduction by moment matching. Foundations and Trends ${ }^{\circledR}$ in Systems and Control, 4(3-4):224-409, 2017. doi: 10.1561/2600000012. 